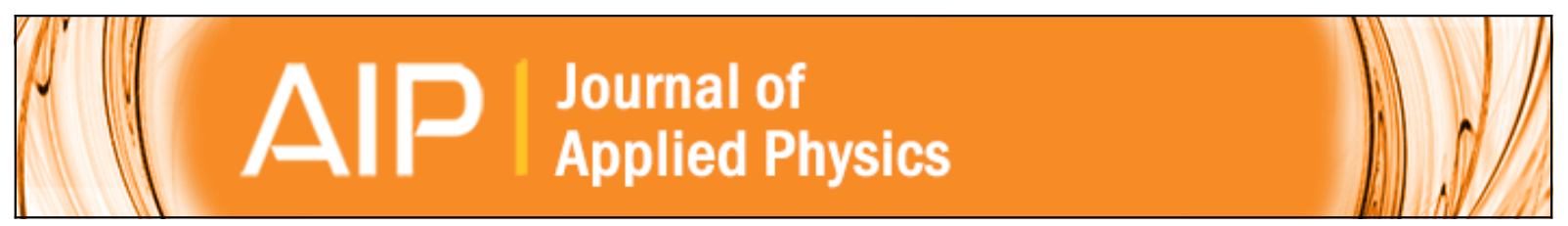

\title{
Laser-driven electron beamlines generated by coupling laser-plasma sources with
} conventional transport systems

P. Antici, A. Bacci, C. Benedetti, E. Chiadroni, M. Ferrario, A. R. Rossi, L. Lancia, M. Migliorati, A. Mostacci, L. Palumbo, and L. Serafini

Citation: Journal of Applied Physics 112, 044902 (2012); doi: 10.1063/1.4740456

View online: http://dx.doi.org/10.1063/1.4740456

View Table of Contents: http://scitation.aip.org/content/aip/journal/jap/112/4?ver=pdfcov

Published by the AIP Publishing

\section{Articles you may be interested in}

Plasma electron source for the generation of wide-aperture pulsed beam at forevacuum pressures

Rev. Sci. Instrum. 84, 023301 (2013); 10.1063/1.4789771

Characterisation of electron beams from laser-driven particle accelerators

AIP Conf. Proc. 1507, 672 (2012); 10.1063/1.4773778

Investigation of a hollow anode with an incorporated ferroelectric plasma source for generation of high-current electron beams

J. Appl. Phys. 94, 6319 (2003); 10.1063/1.1619571

Ultrashort electron bunches generated with high-intensity lasers: Applications to injectors and x-ray sources Appl. Phys. Lett. 83, 3888 (2003); 10.1063/1.1626016

VeryHighBrightness Picosecond Electron Source

AIP Conf. Proc. 680, 1026 (2003); 10.1063/1.1619883

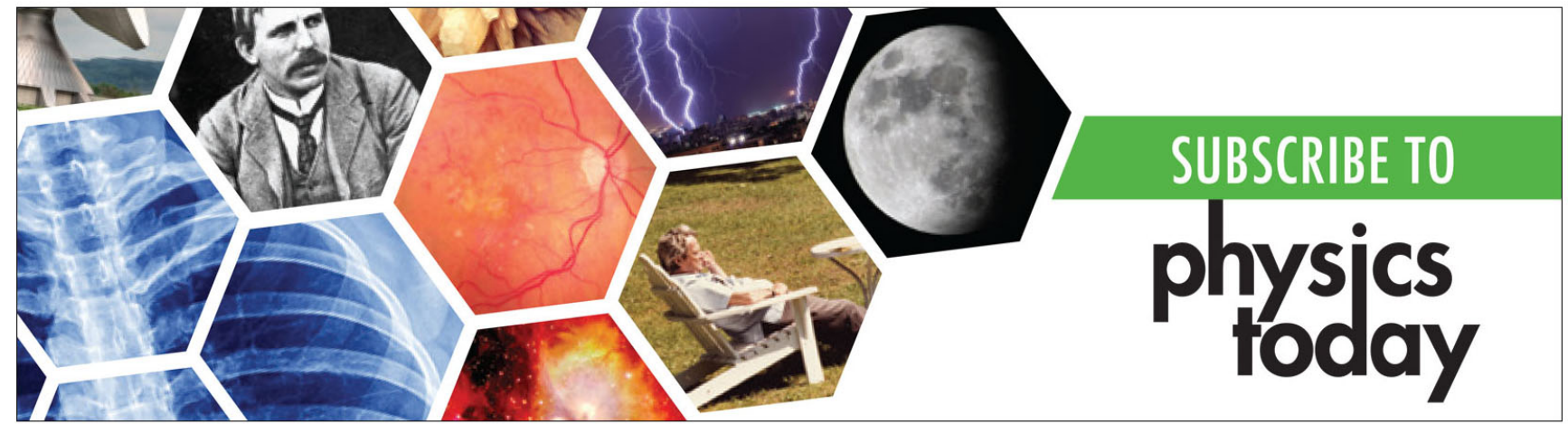




\title{
Laser-driven electron beamlines generated by coupling laser-plasma sources with conventional transport systems
}

\author{
P. Antici, ${ }^{1,2,3, a)}$ A. Bacci, ${ }^{1}$ C. Benedetti, ${ }^{4, b)}$ E. Chiadroni, ${ }^{1}$ M. Ferrario, ${ }^{1}$ A. R. Rossi, ${ }^{1}$ \\ L. Lancia ${ }^{2,3}$ M. Migliorati, ${ }^{2,3}$ A. Mostacci, ${ }^{2,3}$ L. Palumbo, ${ }^{2,3}$ and L. Serafini ${ }^{5}$ \\ ${ }_{1}^{1}$ Istituto Nazionale di Fisica Nucleare (INFN), Laboratori Nazionali di Frascati, Via E. Fermi, 40, \\ 00044 Frascati, Italy \\ ${ }^{2}$ SAPIENZA, University of Rome, Dip. SBAI, Via A. Scarpa 14, 00161 Rome, Italy \\ ${ }^{3} I N F N$ - Sezione di Roma, clo Dipartimento di Fisica - SAPIENZA, University of Rome, P.le Aldo Moro, \\ 2 - 00185 Rome - Italy \\ ${ }^{4}$ University of Bologna and INFN - Bologna, Italy \\ ${ }^{5}$ INFN-Milan and Department of Physics, University of Milan, Via Celoria 16, 20133 Milan, Italy
}

(Received 4 January 2012; accepted 27 June 2012; published online 22 August 2012)

\begin{abstract}
Laser-driven electron beamlines are receiving increasing interest from the particle accelerator community. In particular, the high initial energy, low emittance, and high beam current of the plasma based electron source potentially allow generating much more compact and bright particle accelerators than what conventional accelerator technology can achieve. Using laser-generated particles as injectors for generating beamlines could significantly reduce the size and cost of accelerator facilities. Unfortunately, several features of laser-based particle beams need still to be improved before considering them for particle beamlines and thus enable the use of plasma-driven accelerators for the multiple applications of traditional accelerators. Besides working on the plasma source itself, a promising approach to shape the laser-generated beams is coupling them with conventional accelerator elements in order to benefit from both a versatile electron source and a controllable beam. In this paper, we perform start-to-end simulations to generate laser-driven beamlines using conventional accelerator codes and methodologies. Starting with laser-generated electrons that can be obtained with established multi-hundred TW laser systems, we compare different options to capture and transport the beams. This is performed with the aim of providing beamlines suitable for potential applications, such as free electron lasers. In our approach, we have analyzed which parameters are critical at the source and from there evaluated different ways to overcome these issues using conventional accelerator elements and methods. We show that electron driven beamlines are potentially feasible, but exploiting their full potential requires extensive improvement of the source parameters or innovative technological devices for their transport and capture. (C) 2012 American Institute of Physics. [http://dx.doi.org/10.1063/1.4740456]
\end{abstract}

\section{INTRODUCTION}

Particle accelerators have been one of the most important instruments for scientific research for the past few decades. Since the beginning of particle accelerators, the need for higher beam energies in performing cutting-edge scientific experimentation has pushed the technical progress of accelerator technology, leading to an exponential increase of the beam energy with time. ${ }^{1}$ However, today, technical progress in the area of conventional accelerator technology seems to have come to a point where new technologies should be considered: The conventional acceleration of charged particles relies on the use of radio-frequency (RF) longitudinal electric fields. The energy gain of the charged particles is proportional to the amplitude of the electric fields. Unfortunately, above a threshold field value of some tens of $\mathrm{MV} / \mathrm{m}$, a breakdown occurs, producing a leak of electrons from the metal surface, damaging the resonators, and preventing the particle acceleration. As a consequence, the energy gain per unit

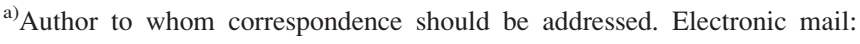
patrizio.antici@uniroma1.it.

b) Present address: LBNL, Berkeley, California 94720-8211, USA.
}

length in conventional accelerators is limited, and high energies can only be reached by additional acceleration modules which yield to very large- and therefore extremely costlyfacilities such as the Large Hardron Collider. ${ }^{2}$

A completely new acceleration technique, which has become possible only in recent years with the advent of high-powered short-pulse laser systems, is the laser-driven plasma acceleration. ${ }^{3}$ In comparison with conventional accelerators, a plasma can generate and withstand electric fields up to TV/m, thus giving it the possibility to accelerate a particle beam to $\mathrm{GeV}$ energies in very short distances. Several experiments to date have demonstrated the effectiveness and efficiency of laser-plasma acceleration, producing particle beams of high quality in the energy range of a few hundred $\mathrm{MeV}$ up to $1 \mathrm{GeV}^{4,5}$ These laser-produced particle beams possess a number of outstanding properties, such as ultra-short pulse duration, high peak currents, and excellent emittance at the plasma-vacuum interface. Both the unique beam properties and the compactness of the acceleration scheme result in strong advantages in terms of size and cost for a high-energy particle accelerating infrastructure.

However, much work still needs to be done in order to produce reliable beamlines based on a laser-plasma source. 
In particular, although the source has its own specific traits, a capture and transport system needs to be implemented for generating a laser-based beamline. In this paper, we present a design of a particle transport system that consists of a laser-driven electron source coupled to conventional magnetic devices (solenoids and quadrupoles) for the capture and transport of the beam. In our approach, we perform a design study aimed at achieving useable laser-driven beams. We use start-to-end simulations for obtaining guidance on which parameters need to be improved and attempt to use the optimized laser-driven beam transport line for a typical application, such as an injector of a free electron laser (FEL).

The paper is organized as follows: Sec. II studies the attributes of laser-generated electrons by the self-injection mechanism in the so-called bubble regime and summarizes the main properties of the beam used as the source for the beamline. Section III analyzes the normalized emittance effects for beams with high energy spread and high divergence. Potential transport lines for laser-driven beamlines are discussed in Secs. IV-VI, while Sec. VII shows the limitations of using those beams in conventional undulators for the generation of FEL radiation.

\section{LASER-GENERATED ELECTRON BEAMS}

There are many different mechanisms for laser-plasma acceleration of electrons. In our study, we will focus on electrons produced in the bubble regime ${ }^{6,7}$ which occurs when the laser waist $w_{0}$ is smaller than the plasma wavelength $\lambda_{p}$ and the dimensionless laser parameter $a_{0}$, defined as $a_{0}=e E /(m \omega c)$, is sufficiently high (e.g., $a_{0} \approx 2.5-3$ ). Nevertheless, our conclusions will be valid also for most other experimental setups, such as laser wakefield acceleration (LWFA) in a mildly nonlinear regime or plasma wakefield accelerators.

When a high power laser $\left(I \geq 10^{18} \mathrm{~W} / \mathrm{cm}^{2}\right)$ impinges on an under-dense plasma, the ponderomotive force pushes the electrons away from the plasma, creating a bubble-shaped volume almost completely depleted of electrons (also called the bubble). Due to the presence of the background positive ions (assumed to be motionless), strong electric fields are present with a peak longitudinal value that scales as

$$
E_{z}[\mathrm{~V} / \mathrm{m}] \approx 100 \sqrt{n_{0}\left[\mathrm{~cm}^{-3}\right] a_{0}},
$$

where $n_{0}$ is the plasma density. Such a scaling law assumes that the bubble is stable, spherical, and matches the optimum conditions for the laser parameters. In this case, the peak value for the longitudinal force (responsible for the electron acceleration) is of the same order of magnitude as its transverse component which gives the focusing behavior. ${ }^{3}$ This transverse component is much more pronounced than in conventional accelerators. Eventually, some electrons are injected into the strongly accelerating longitudinal field at the bubble bottom; since the detailed injection mechanism is highly nonlinear (and not yet completely understood), it is hard to finely control it. Electron acceleration can occur as a single event (which is typical for some regimes ${ }^{8,9}$ ), or in a pulsed, partly modulated process as considered in the present case. There are also some experimental schemes where injection can be partially controlled by a counter propagating laser ${ }^{10}$ although, in that case, the injected charge is very low. Recently, schemes where injection is induced by tailoring the plasma longitudinal density have been tried. ${ }^{11}$

If at the beginning of the acceleration process the plasma mean energy (temperature) is assumed to be very low (cold), the transverse average normalized electrons momentum at the injection into the bubble can reach magnitudes in the order of $a_{0}$. Only electrons with a longitudinal component of the velocity greater than the bubble speed will be captured and accelerated; therefore, plasma electrons injected into the bubble are quite energetic (hot).

Such electrons also possess a transverse (thermal) momentum of the same order of magnitude of the longitudinal one, which corresponds to a few $\mathrm{MeV} / \mathrm{c}$ in our numerical simulations. Typical plasma accelerated beams (considered in this paper) reach energies in the order of the $\mathrm{GeV}$ and therefore their divergence approaches a few mrad when they exit from the plasma. For comparison, typical electrons produced in a RF photoinjector possess a transverse momentum of few $\mathrm{eV} / \mathrm{c}$ when extracted from the photocathode; the subsequent acceleration brings the beam to the energy of a few $\mathrm{MeV}$ and the divergence down to $10^{-3} \mathrm{mrad}$. Hence, in laser-driven accelerators, the relativistic momentum at injection into the bubble is responsible for a high divergence and a large uncorrelated energy spread, as compared to bunches in conventional accelerators. Moreover, since the injection occurs along a long plasma channel (typically a few millimeters), the very high field gradient increases the effect of a large correlated energy spread and the lack of divergence reduction deeply influences the behavior of the beam as soon as it leaves the plasma channel. The presence of strong focusing fields implies that the beam is emittance-dominated; its transverse distribution, both in charge density and in momentum, is expected to be well represented by a Gaussian curve. From the transverse envelope equation, after the plasma-vacuum interface, we can expect that the beam transverse size would increase, due to free diffraction, as $^{12}$ :

$$
\sigma_{x}(z)=\sqrt{\sigma_{0}^{2}+2 \sigma_{0} \sigma_{0}^{\prime} z+\left(\frac{\varepsilon^{2}}{\sigma_{0}^{2}}+\sigma_{0}^{\prime 2}\right) z^{2}},
$$

where $z$ is the longitudinal coordinate, $\varepsilon$ the geometric r.m.s. emittance, $\sigma_{0} \equiv \sigma_{x}(0)$ the initial transverse size, and $\sigma_{0}^{\prime} \equiv$ $d \sigma_{x} / d z(0)$ its first derivative.

For the beam transport system, we will focus on the capture and transport of bunches generated by $100 \mathrm{TW}$ class lasers, which are currently being established worldwide in different laboratories. These lasers are used as test facilities for larger scale infrastructures that are currently being planned or already under construction, such as 1-10 PW lasers. We have used the 3D-particle in cell (PIC) code ALaDyn (Ref. 15) for simulating the laser-matter interaction. The advantage of using simulated particle beams is that they provide more details about the beam properties (3D velocity of each particle, etc). We have verified that the results are 
TABLE I. Main beam parameters used for the design of the electron beam transport. The beam is assumed to be with cylindrical geometry, only the $x$ transverse coordinate is considered.

\begin{tabular}{lc}
\hline \hline Charge & $700 \mathrm{pC}$ \\
Energy & $910 \mathrm{MeV}$ \\
Energy spread, $\sigma_{\varepsilon}$ & $6.4 \%$ \\
Bunch length & $2 \mu \mathrm{m}$ \\
Transverse size, $\sigma_{x}$ & $0.5 \mu \mathrm{m}$ \\
Transverse divergence, $\sigma_{x \prime}$ & $3 \mathrm{mrad}$ \\
Emittance, $\varepsilon$ & $1.4 \times 10^{-3} \mathrm{~mm} \mathrm{mrad}$ \\
Normalized emittance, $\varepsilon_{n}$ & $2.5 \mathrm{~mm} \mathrm{mrad}$ \\
\hline
\end{tabular}

consistent with experimental values. The following parameters have been used as input for the simulations: laser with wavelength $\lambda=0.8 \mu \mathrm{m}$, contrast ratio of $10^{10}$, pulse duration (FWHM) $\tau=30 \mathrm{fs}$, and waist $=15.5 \mu \mathrm{m}$, delivering an intensity of $I=5 \times 10^{19} \mathrm{~W} / \mathrm{cm}^{2}$, similar to what is currently obtained on 200 TW class lasers such as the FLAME laser, currently being used at the Frascati National Laboratories. ${ }^{13}$ For the plasma, we have considered a plasma of length $4.1 \mathrm{~mm}$ and electron density $3 \times 10^{18} \mathrm{~cm}^{-3}$ with plasma down ramp length of $100 \mu \mathrm{m}$. These parameters were chosen since, from scaling laws, they shall produce the highest electron beam energy with the available laser power. ${ }^{14} \mathrm{We}$ are aware that additional emittance growth can also be generated by the plasma down ramp length, as reported by Kneip et $a .^{28}$ and Sears et al. ${ }^{9}$ : In our simulations, the plasma down ramp length was $100 \mu \mathrm{m}$ long, much shorter than the betatron wavelength. A proper tailoring of the plasma down ramp would produce an adiabatic defocusing of the bunch (this means that an increase of the transverse beam size would result in a decrease of the divergence keeping the emittance constant) and prevent a rapid normalized emittance dilution. However, this process would not affect the energy spread and consequently our study of matching the entire bunch to a transport line.

The electron parameters obtained are reported in Table I, while the longitudinal phase space bunch distribution is shown in Fig. 1. Concerning the transverse phase space, the

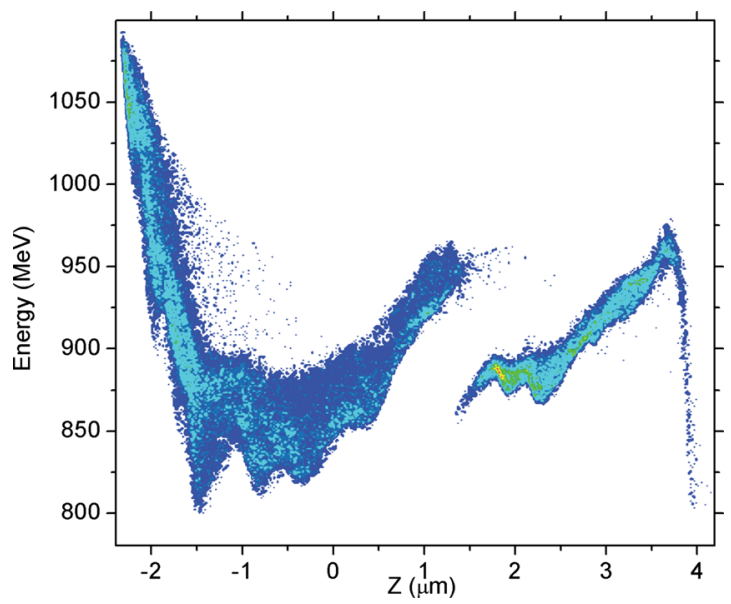

FIG. 1. Longitudinal phase space distribution obtained with the PIC code ALaDyn and used for the laser-driven electron beamline. beam distribution appears almost Gaussian in the transverse position and divergence, as expected for an emittancedominated beam; at the plasma exit, the beam is in a waist and its position and divergence are uncorrelated.

\section{HIGH DIVERGENCE AND HIGH ENERGY SPREAD BUNCHES}

In order to be transported in a conventional accelerator lattice structure, the electron bunch needs to be matched to the transport beamline.

Once the beam comes out from the laser-plasma interaction region, it suddenly passes from the extremely intense focusing fields inside the bubble to free space. A possible way to capture and control the beam is using strong focusing magnetic fields (i.e., using quadrupoles or solenoids), as close as possible to the interaction point. However, from the parameters of Table I, the Twiss beta function, $\beta_{T}=\sigma_{x}^{2} / \varepsilon$, is as low as a fraction of $\mu \mathrm{m}$, many orders of magnitude lower than what is generally reachable in conventional accelerators.

Moreover, the transverse beam correlation, initially zero, increases quadratically with the distance becoming very rapidly the predominant term in the emittance due to the relative high divergence. Also, the transverse dimension of the beam shows a constant growth rate that becomes very difficult to control by conventional magnets that do not have enough strength to counteract such beam behavior: After $1 \mathrm{~cm}$ (a minimum distance to adopt between the laserinteraction point and a coupling device), the transverse size is about 60 times higher than the initial value. In addition to the beam's strong growth in size, the drift space also generates additional beam degradation by greatly increasing its normalized emittance. ${ }^{32}$ This can be seen in Fig. 2 that shows the transverse normalized emittance in the $1 \mathrm{~cm}$ drift space following the interaction region, obtained by using the code TSTEP, ${ }^{16}$ a derivative of PARMELA (Ref. 17), and a well established macroparticle code in the conventional accelerator community.

If space charge and wakefield effects are neglected, as in this case, one would expect the emittance to remain constant in a free drift. This is valid for the geometric emittance $\varepsilon$, since the transverse motion is uncoupled from other planes, and it is generally valid also for the normalized emittance $\varepsilon_{n}$. In fact, a measure of the normalized emittance $\varepsilon_{n}$ is commonly obtained, in conventional accelerators, by

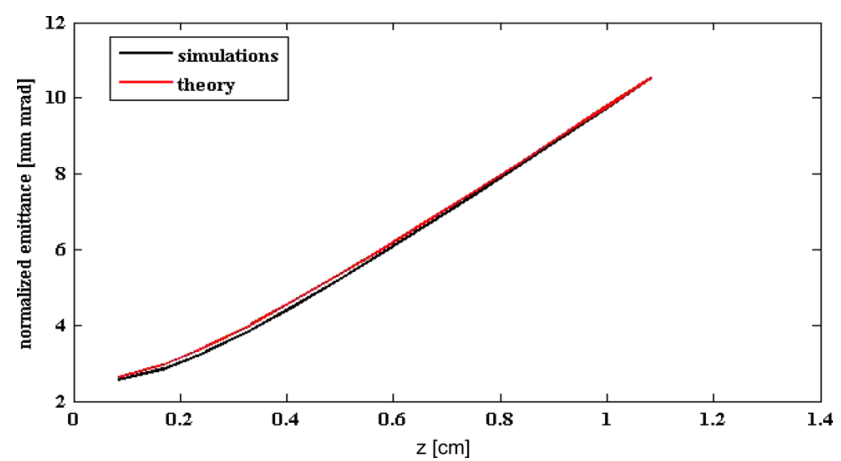

FIG. 2. Transverse normalized emittance along a drift of $1 \mathrm{~cm}$ : theory (red line) and simulation (black line). 
measuring separately $\varepsilon$ and the average relativistic factor $\langle\gamma\rangle$ and setting $\varepsilon_{n}=\langle\gamma\rangle \varepsilon$. This approach for calculating normalized emittance in laser-plasma generated electron beams has been used in Refs. 8, 9, 18, 28, and 29. A more detailed analysis of the definition of the normalized emittance shows that this approximation does not hold for laser-driven electron beams.

In general, we define $\varepsilon_{n}$ as:

$$
\varepsilon_{n}^{2}=\left\langle x^{2}\right\rangle\left\langle\beta^{2} \gamma^{2} x^{\prime 2}\right\rangle-\left\langle x \beta \gamma x^{\prime}\right\rangle^{2}
$$

with $\beta=v / c$ and $\gamma$ the particle relativistic factor, and $x$ and $x /$ the transverse position and its divergence, respectively. If the correlation between energy and transverse position is negligible, as in a drift without collective effects, we can write

$$
\varepsilon_{n}^{2}=\left\langle\beta^{2} \gamma^{2}\right\rangle\left\langle x^{2}\right\rangle\left\langle x^{\prime 2}\right\rangle-\langle\beta \gamma\rangle^{2}\left\langle x x^{\prime}\right\rangle^{2}
$$

The definition of the relative energy spread $\sigma_{E}$ reads

$$
\sigma_{E}^{2}=\frac{\left\langle\beta^{2} \gamma^{2}\right\rangle-\langle\beta \gamma\rangle^{2}}{\langle\gamma\rangle^{2}}
$$

which can be inserted in Eq. (4) to give

$$
\varepsilon_{n}^{2}=\langle\gamma\rangle^{2} \sigma_{E}^{2}\left\langle x^{2}\right\rangle\left\langle x^{\prime 2}\right\rangle+\langle\beta \gamma\rangle^{2}\left(\left\langle x^{2}\right\rangle\left\langle x^{\prime 2}\right\rangle-\left\langle x x^{\prime}\right\rangle^{2}\right)
$$

Assuming relativistic electrons $(\beta=1)$, we get

$$
\varepsilon_{n}^{2}=\langle\gamma\rangle^{2}\left(\sigma_{E}^{2} \sigma_{x}^{2} \sigma_{x^{\prime}}^{2}+\varepsilon^{2}\right)
$$

If the first term on the right hand side is negligible, the normalized emittance reduces to the usual expression $\langle\gamma\rangle \varepsilon$. At the plasma-vacuum interface, the beam is in a waist, thus one can write in Eq. (2) $\sigma_{0}^{\prime}=0$ and $\varepsilon^{2} / \sigma_{0}^{2}=\sigma_{x^{\prime}}^{2}$. In the limit $s \gg 1$, Eq. (2) reads $\sigma_{x}(s) \approx \sigma_{x^{\prime}} s$ and Eq. (7) becomes

$$
\varepsilon_{n}^{2} \approx\langle\gamma\rangle^{2}\left(\sigma_{E}^{2} \sigma_{x^{\prime}}^{4} s^{2}+\varepsilon^{2}\right)
$$

The last expression is represented in Fig. 2 by the red curve, which shows a very good agreement with the results of TSTEP. Note that for protons and heavier ionic species, the assumption of relativistic motion done in deriving Eq. (7) is usually not valid; this worsens the effect on normalized emittance and produces a lengthening of the produced bunch.

Such rapid emittance dilution is linked to the fact that the betatron frequency of a beam critically depends on its energy. During the drift, each chromatic component rotates with its own velocity in the transverse phase space, spreading out the area occupied by the whole bunch; the resulting projected normalized emittance then becomes a function of both position and energy spread. This is shown in Fig. 3 (up) where we can see a spectrum of the bunch in which a few (arbitrarily chosen) energetic components (bins) have been outlined using different colors: The chromatic effect is depicted in Fig. 3 (down) where the transverse phase space is reproduced after removing the $x-p_{x}$ correlation. To high-

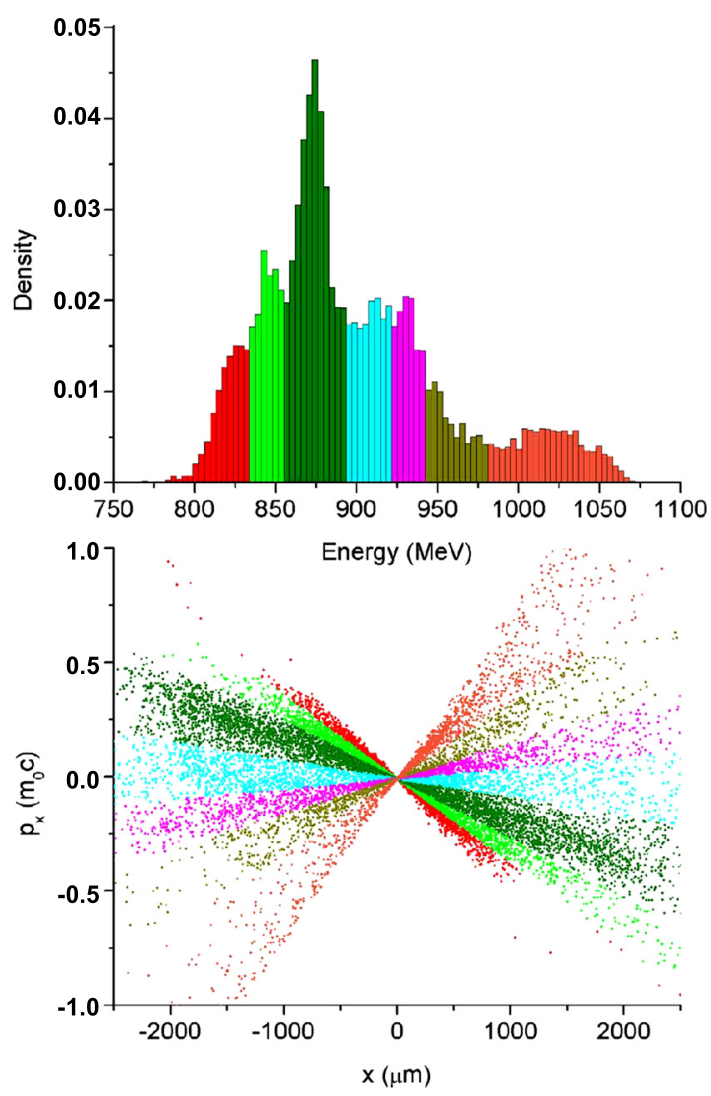

FIG. 3. Bunch spectrum (up) and decorrelated transverse phase space (down). A free drift space of $1 \mathrm{~m}$ has been chosen to enhance the chromatic effects. In the upper plot, the ordinate in the histogram indicates the amount of particles normalized to all particles.

light the effect, the bunch spectrum and phase space have been taken $1 \mathrm{~m}$ after interaction. Indeed, if the divergence $x^{\prime}$ is defined as $x^{\prime} \approx p_{x} /\langle p\rangle$ (see, for example, Ref. 12), we clearly see that an emittance dilution is not detected (see Fig. 3).

Equation (7) can also be used to predict the normalized emittance behavior in the presence of magnetic fields, like quadrupoles for example, when there is some coupling between energy and the transverse coordinate.

Even if the agreement between theory and simulation is worse than in the case of a simple drift, Eq. (7) remains a valid analytical tool to quickly determine the behavior of the normalized emittance in a transport line without running macroparticle tracking codes.

Additionally, chromatic effects could be different for normalized slice emittance, which is the critical parameter for some applications; for example, in FELs the longitudinal slices (i.e., the length of the projection of particles considered) are of the order of the cooperation length. If such slices are sufficiently thin, the contribution of the correlated energy spread to the slice emittance is usually negligible with respect to uncorrelated energy spread (which depends on thermal emittance). Therefore, with regard to the slice parameters, laser-plasma beams may not differ much from beams produced by conventional accelerators.

The results presented above demonstrate that lasergenerated electron beams have a different behavior than 
conventional accelerator beams. In a drift immediately following the laser-plasma interaction point, we find strong normalized emittance growth (up $1000 \mathrm{~mm} \mathrm{mrad} / \mathrm{m}$ ) depending on energy spread, beam divergence, and beam size. Matching such a beam to a transport line, without worsening the bunch normalized emittance, requires the optical elements to have a focal length of the order of the bunch $\beta$ function, which is impossible to achieve with conventional technology. Moreover, these elements produce effects which are energy dependent so that focusing chromatic effects would surely manifest; an achromatic focusing device is not able to be realized by magnetic quadrupoles or solenoids. ${ }^{20}$

\section{QUADRUPOLE MATCHING LINE}

Despite these issues, we have investigated two ways for controlling and matching the beam to a focusing-defocusing (FODO) transport line, using quadrupoles or solenoids. A quadrupole matching line is more commonly used in conventional particle accelerators due to its lower cost as compared to a solenoid line. For the design of our matching system, we have first used the code TRACE $3 \mathrm{D},{ }^{21}$ an interactive beam dynamics program that calculates the envelopes of a bunched beam, including linear space charge forces, through a userdefined transport system. Once the beam line has been optimized, the parameters of the magnets have been inserted in the tracking code TSTEP to account for the beam distribution function.

In Fig. 4, we show the optimized beam line obtained with TRACE 3D. Starting from very low Twiss $\beta_{T}$ values, of the order of $0.2 \mathrm{~mm}$, by using a matching system of three quadrupoles (triplet) with a total length of $174 \mathrm{~mm}$, we manage to obtain at the end of the transport line a bunch with Twiss parameters $\alpha_{T}=0$ and $\beta_{T}=45 \mathrm{~m}$. Such final beam parameters can be transported with a periodic FODO system without specific problems. Also the transverse dimensions of the bunch allow a conventional transport after the matching line.

For a deeper investigation of the beam dynamics, we have introduced the magnet's parameters and dimensions into the code TSTEP. The bunch remains confined below $0.4 \mathrm{~mm}$ and the behavior agrees with the results of TRACE 3D. Even if the quadrupole matching line is quite attractive, due to its simplicity and affordability, two problems arise by using this kind of transport system: The first one being purely technological and the second related to the definition

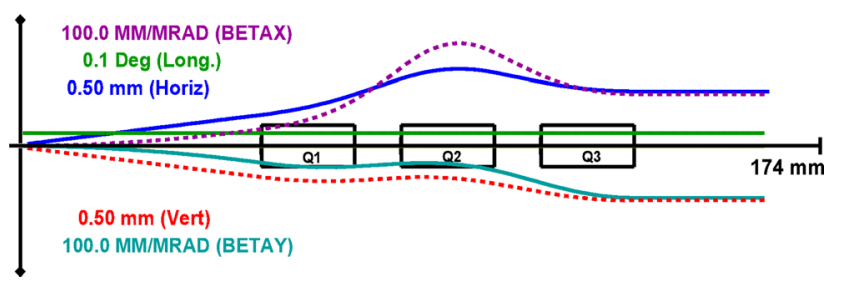

FIG. 4. Matching transport line with quadrupoles as optimized using the TRACE 3D code. Axis limits for the different parameters are indicated by the normalized ordinate in figure.

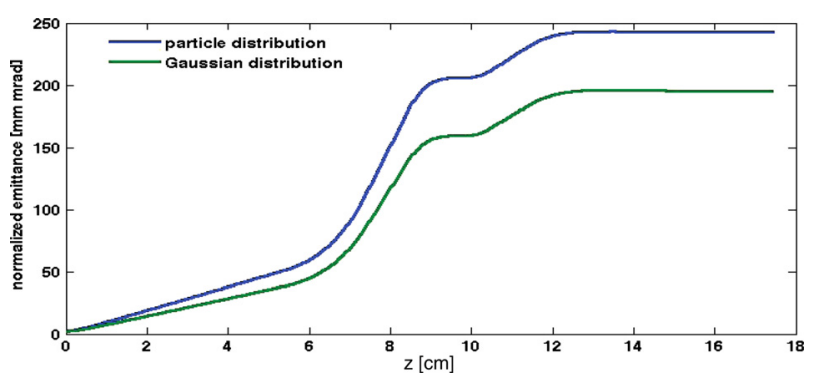

FIG. 5. Transverse normalized emittance along the quadrupole matching line obtained with TSTEP.

of normalized emittance. Concerning the first problem, simulations show that for a beam control, the strength of the quadrupole gradient necessary to contain the divergence of such beams reaches values as high as $5000 \mathrm{~T} / \mathrm{m}$, a factor 10 higher than the maximum gradient currently available and obtained using permanent magnet quadrupoles. ${ }^{22}$ Reducing the quadrupole gradient in the simulation to values of 500 $\mathrm{T} / \mathrm{m}$, which is considered state-of-the-art, and even increasing the number of quadrupoles, we did not manage to control the beam explosion. The second problem is related to the normalized emittance $\varepsilon_{n}$. Using the quadrupole matching line that has been optimized for the optics, even if we allow the presence of permanent magnet quadrupoles with gradients of $5000 \mathrm{~T} / \mathrm{m}$, we obtain a normalized emittance at the exit of the beamline reaching up to $250 \mathrm{~mm} \mathrm{mrad}$, excessively high for an accelerator. In Fig. 5, we compare the results of the simulations obtained with TSTEP starting from two different electron beam parameter cases: In the first case, we used the electron distribution shown in Fig. 1 (blue line). In the second case, we took mean values of the beam parameters and generated the particle distribution using an ideal Gaussian behaviour (green line). As can be seen, both cases show a similar trend, which indicates that the problem is not linked to the peculiarity of the beam distribution, but to the mean initial beam parameters of the laser-driven beam.

\section{SOLENOID MATCHING LINE}

An alternative to focusing with quadrupoles is the use of solenoids. These devices, acting over a longer distance than quadrupoles, allow a smoother control of the bunch transverse size. As in Sec. IV, we have first optimized the beam line using TRACE 3D. A solenoid of about $20 \mathrm{~cm}$, located very close to the laser-plasma interaction point, is able to control the beam size and the Twiss beta function. At the exit of the solenoid we obtain a beta function of about $88 \mathrm{~m}$ in both directions, and a transversely uncorrelated bunch $\left(\alpha_{T}=0\right)$.

We have then performed simulations with TSTEP, obtaining a transverse size behavior similar to that obtained using quadrupoles, with a maximum transverse envelope remaining confined below $0.4 \mathrm{~mm}$.

A transport line using solenoids is more expensive and complex than one using quadrupoles. However, in this case, the intensity of magnetic fields inside the solenoid, even though extremely high, seems to be within reach of the 
actual state-of-the-art technology. In fact, the optimization with TRACE 3D requires a longitudinal magnetic field of about $45-50 \mathrm{~T}$, an unusual value for conventional accelerators. As an example, for the SPARC (Refs. 19 and 23) and LCLS (Ref. 24) photoinjectors the magnetic field is below $0.5 \mathrm{~T}$. High field solenoids have been under investigation using high temperature superconductors, and a conceptual design of a solenoid of $45 \mathrm{~T}$ has been recently presented. ${ }^{25}$ As expected, by using solenoids the normalized beam emittance endures the same effect of uncontrollable increase as the one shown in Fig. 5. At the end of the beamline it reaches a value of about $200 \mathrm{~mm} \mathrm{mrad}$, not much different from the result with quadrupoles.

Even though a transport system using a strong solenoidal field seems feasible from the optics point of view, the problem of an uncontrollable increase of the normalized emittance still affects these laser-plasma generated bunches due to the combination of high energy spread and divergence, and conventional magnet transport systems are not able to counteract this effect. We can conclude that in order to contrast the phenomenon it is necessary to act directly on the laser-plasma source and improve the beam qualities.

\section{CONVENTIONAL STRATEGIES TO IMPROVE BEAM TRANSPORT}

The beam current obtained by laser-driven electrons (Table I) shows however the advantage of reaching $\approx 100 \mathrm{kA}$, a factor between 100 and 1000 times higher than what required in photoinjectors for driving a FEL. One could therefore cut part of the initial distribution, reducing the beam divergence and the energy spread at the cost of losing some charge. The simplest way to do that is by using slits or apertures, placed in optimized points of the matching line, which cut transversely the beam.

After several optimizations and for our beam parameters, a good choice is to position a first rectangular aperture at $60 \mathrm{~cm}$ from the laser-plasma interaction point, where the longitudinal magnetic field is zero. The aperture cuts the bunch horizontally at $\pm 0.4 \mathrm{~mm}$ and vertically at $\pm 0.3 \mathrm{~mm}$. In this way, we pass from the initial charge of $700 \mathrm{pC}$ to about $230 \mathrm{pC}$. The initial current is reduced to about $40 \mathrm{kA}$, still a very high current, with the advantage that the normalized emittance is strongly reduced by more than a factor 7 . However, the energy spread is only little reduced, since it passes from $6.4 \%$ to $6 \%$. It is interesting to note that in this

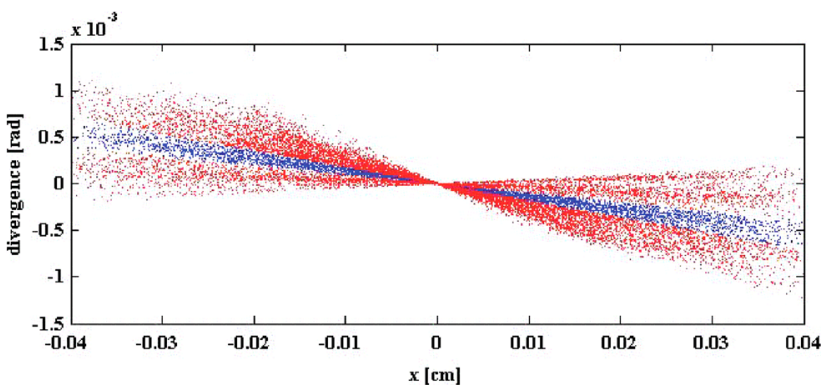

FIG. 6. Transverse beam distribution obtained with TSTEP after the cut with the rectangular aperture: blue dots represent particles having energy spread less than $2 \%$, red dots having a larger one.

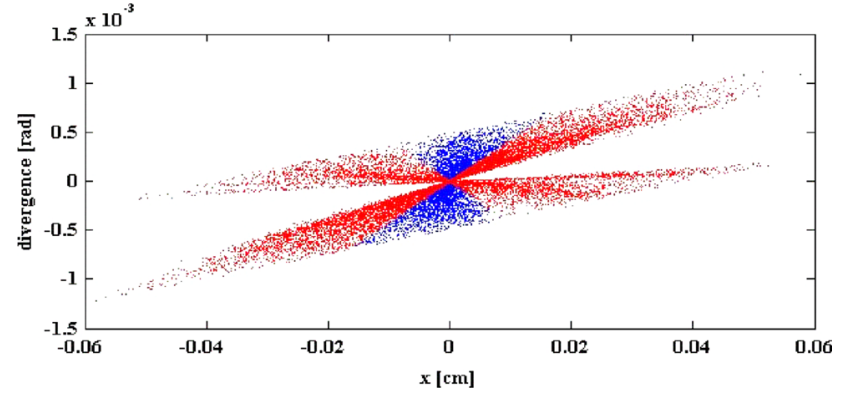

FIG. 7. Transverse beam distribution obtained with TSTEP at a distance of $140 \mathrm{~cm}$ from the laser-plasma interaction, with blue dots representing particles having energy spread less than $2 \%$, red dots having a larger one.

position there is a profitable correlation between energy and transverse phase space. In Fig. 6, we show the transverse phase space after the cut with the blue dots representing all particles whose energy differs less than $2 \%$ from the initial mean energy and with the red dots all the others.

Since in a drift all the particles having a divergence greater than zero move in the right direction of the figure, while particles with negative divergence move toward left, the bunch rotates in the phase space, enlarging it: A cut at the correct position allows reducing the energy spread. The same phase space after an additional drift of $80 \mathrm{~cm}$ (i.e., at $140 \mathrm{~cm}$ from the laser-plasma interaction point) is shown in Fig. 7. Appropriately positioning a rectangular aperture allows cutting particles having a high energy spread.

With an aperture of dimensions $80 \mu \mathrm{m}$ width and $60 \mu \mathrm{m}$ height, we reduce the energy spread to $2.74 \%$, keeping a charge of about $70 \mathrm{pC}$, and a peak current of $13.5 \mathrm{kA}$. Such a bunch has characteristics close to those of conventional accelerators and is suitable to be captured by a FODO periodic line, since the Twiss beta-functions are $\approx 20 \mathrm{~cm}$. This matching line gives a normalized emittance of about $10 \mathrm{~mm}$ $\mathrm{mrad}$, a factor 40 lower than what was obtained without cuts. Moreover, the transverse cuts are quite easy to realize with simple rectangular apertures and, despite the particle loss, we reach an acceptable high current.

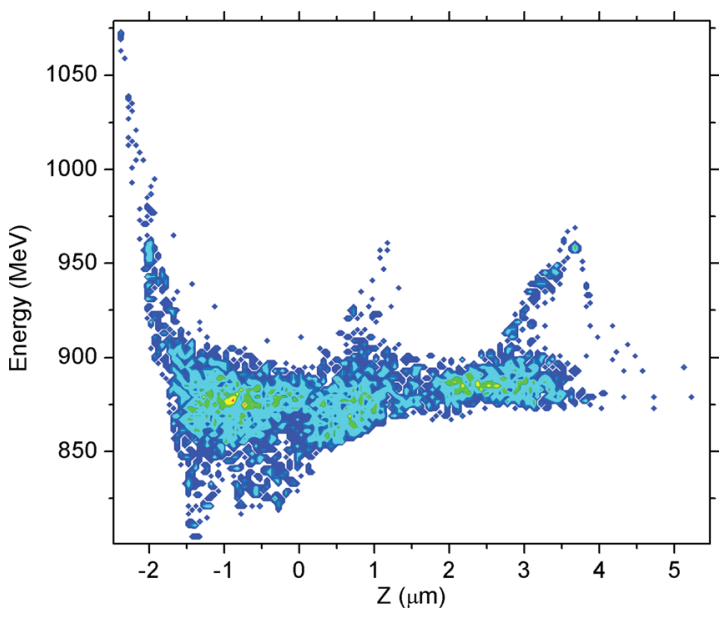

FIG. 8. Longitudinal phase space particle distribution obtained with TSTEP after having passed through two transverse rectangular apertures selecting part of the beam in order to reduce the energy spread. 
Unfortunately, even with using apertures, the energy spread cannot be strongly reduced to values less than $1 \%-$ $2 \%$ without a consistent loss of current. If we want to maintain a high current, other mechanisms should be devised, e.g., a transport through a bending magnet or an isochronous system. In Fig. 8, shows the longitudinal phase space particle distribution after the second aperture; particles with high energy $(\geq 1 \mathrm{GeV})$, far from the core of the bunch, give a high contribution to the energy spread but only add minimal beam current.

Particles with such an energy distribution cannot be tailored using simple transverse apertures but need first to pass through a dispersion region, realized with bending magnets that produce energy dependent transverse trajectories. These magnets can be exploited to create a transverse beam-energy correlation that allows to properly cut the energy distribution. However, the good beam quality is worsened as soon as the beam enters in a bending magnet, making it again hard to control the bunch and to keep its good characteristics. Moreover, given the high current, coherent synchrotron radiation can generate microbunching.

Additional difficulties for the implementation of such a capturing system come from the shot-to-shot fluctuations, typical for high-power laser systems. These laser fluctuations, but also inhomogeneities in the plasma density, reflect on the electron acceleration and the electron beam pointing. For similar laser-interaction conditions as we have assumed, these pointing stability is in the order of the mrad. ${ }^{30}$

Due to the small apertures dimensions (e.g., a width of $80 \mu \mathrm{m}$ ), a deviation from the nominal beam axis could easily yield to a complete beam loss. An alternative strategy for overcoming this issue could be tuning the transport line on a well defined energy zone, e.g., the neatly defined energy peak seen on the spectrum in Fig. 3 around $850 \mathrm{MeV}$. We expect that the electrons with higher or lower energies, being unmatched, would be lost in the beampipe system (hitting the walls), and globally the transport would produce a beam with low energy spread, not significantly affected by chromatic effects. However, this scheme can also fail, due to the same limitations on stability and repeatability, since the neat peak energy inside the bunch, its central energy, and the mean energy of the entire bunch are still all strongly affected by the same laser-jitter effects, typical of high-power lasers and laser-plasma interaction. From the above discussion, we can conclude that a great amount of work needs to be done on the stability and repeatability of plasma generated beams

TABLE II. Average beam parameters used for the SASE FEL simulation.

\begin{tabular}{lc}
\hline \hline Electron charge, $Q$ & $72 \mathrm{pC}$ \\
Beam energy & $881 \mathrm{MeV}$ \\
Energy spread (rms) & $2.74 \%$ \\
Bunch length $(\mathrm{rms}), \sigma_{\tau}$ & $1.6 \mu \mathrm{m}$ \\
Average current, $Q /\left(\sqrt{2 \pi} \sigma_{\tau}\right)$ & $5.4 \mathrm{kA}$ \\
$\sigma_{x}, \sigma_{y}$ & $33,27 \mu \mathrm{m}$ \\
$\sigma_{x^{\prime}}, \sigma_{y^{\prime}}$ & $0.2 \mathrm{mrad}$ \\
$\varepsilon_{n x}, \varepsilon_{n y}$ & $12.8 \mathrm{~mm} \mathrm{mrad}$ \\
\hline \hline
\end{tabular}

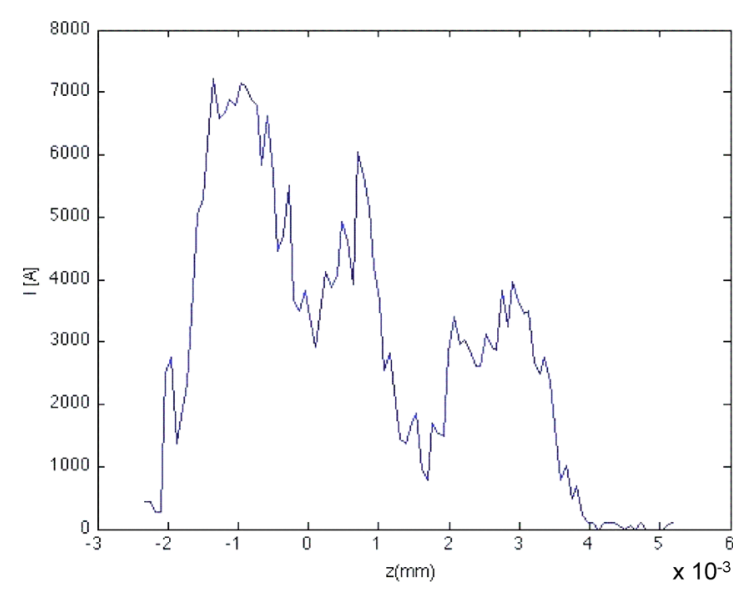

FIG. 9. Beam current profile at the entrance of the undulator section.

before those beams can be used to obtain a quality compatible with a conventional accelerator transport line.

\section{APPLICATION TO FREE ELECTRON LASERS}

In order to assess the potentiality of a laser-plasma generated beam for typical applications, we have numerically studied the propagation of such a beam into undulators for the generation of hard $\mathrm{x}$-rays; in the following, we deal with the beam after the matching discussed in Sec. VI. The simulations of the output characteristics of the SASE FEL radiation, such as temporal and spectral profiles, have been performed by means of the time dependent threedimensional simulation code GENESIS $1.3{ }^{26}$ accounting for slippage, diffraction, emittance, and energy spread effects. For the undulator, we have used the typical parameters as given in the SPARX hard x-ray undulator beamline (undulator period and RMS parameter, $\lambda_{u}=1.5 \mathrm{~cm}$ and $K_{R M S}=0.907$, respectively), ${ }^{27}$ in order to produce SASE FEL radiation at $4.6 \mathrm{~nm}$ at the fundamental wavelength. The electron beam average parameters are reported in Table II and the beam current profile is shown in Fig. 9.

Again we treat two cases for simulating the saturation power. In the first case, we used the mean values reported in Table II and a Gaussian current distribution (see Fig. 10). In the second case, we used the actual bunch distribution obtained by the TSTEP simulation (see Figure 10). For the

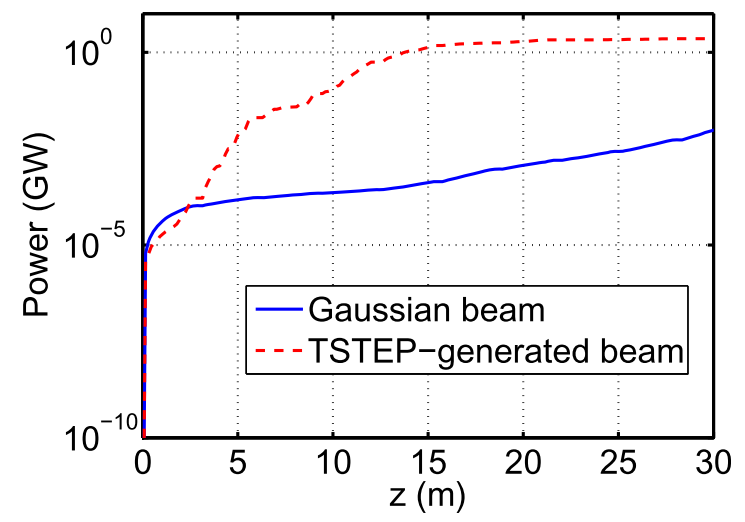

FIG. 10. Power contained in the pulse radiation along the undulator for the TSTEP-generated beam distribution and for the Gaussian bunch case. 


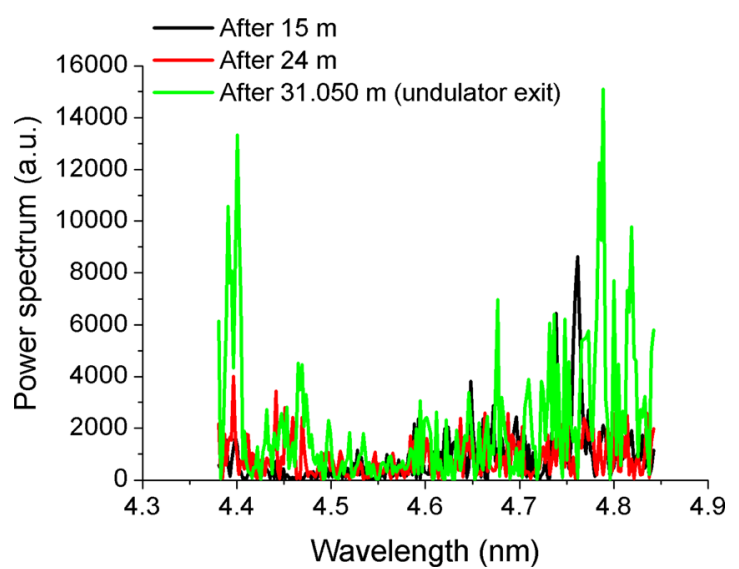

FIG. 11. Power spectrum of a $4.6 \mathrm{~nm}$ radiation generated by the hard $\mathrm{x}$-ray undulator beamline at saturation $(31 \mathrm{~m})$ using as input the distribution obtained at the exit of the beamline by the code TSTEP.

Gaussian bunch, the saturation length is much longer than the undulator length $(31 \mathrm{~m})$, while, in the case using the simulated bunch distribution, the onset of saturation is evident already after $15 \mathrm{~m}$ (being the $3 \mathrm{D}$ gain length about $0.7 \mathrm{~m}$ ). This is due to the fact that in the Gaussian bunch case the bunch slices have a higher current, and therefore it takes more time to reach saturation. In the simulation, we note that the peak current decreases along the undulator because of particle losses due to both, a not well optimized matching at the undulator entrance, and a large beam divergence. Therefore, the current of the bunch contributing to the SASE FEL process is much smaller than the one shown in Fig. 9; we would have expected an even smaller saturation length if the current distribution remained constant along the undulator and equal to Fig. 9. The issue of such beams is that the excellent properties at the source (e.g., brilliance) cannot be fully exploited because of the difficulties in matching them to the undulator line. This has already been experimentally acknowledged by Fuchs et al. ${ }^{31}$ In their experiment, in which laser-driven electrons were shaped using conventional accelerator elements in order to adapt them to the undulators, the conventional accelerating elements were acting only on the part of the phase space that was adapted to the element and that could be considered as a conventional beam. Therefore, more than shaping the beam, these elements are selecting a portion of the total charge, which corresponds to the portion within the phase space in which the electron population has an acceptable energy spread to be transported with the conventional accelerator elements. In their case, the transported current was $10 \%$ of the total, i.e. only a small fraction.

The spectral analysis for the $4.6 \mathrm{~nm}$ radiation case and using the TSTEP parameters is shown in Fig. 11. Three different cases are considered: at the saturation $(15 \mathrm{~m}$, black curve), after $24 \mathrm{~m}$ (red curve), and at the end of the undulator (green curve). The emission is far from being monochromatic and it is centered around the fundamental wavelength, with a $3 \%$ radiation bandwidth spread.

Our simulations show that, despite a large emittance and huge energy spread, the high peak current allows for SASE FEL saturation, even within moderate undulator lengths and a significant loss of the bunch charge during its propagation in the undulator. However, the radiation spectrum has not the typical shape of conventional SASE FELs (showing spike radiation) because the energy spread of the beam is still too high (even higher than the FEL $\rho$ parameter).

\section{CONCLUSIONS}

In this paper, we have shown that a laser-plasma generated electron beam possess unique characteristics that require a different approach when handling them for generating accelerator beamlines. In particular, the combination of their extremely high initial energy, their large divergence, and pronounced energy spread makes them a challenging source for capture and transport. Conventional accelerator elements, such as quadrupoles and solenoids, can be used for shaping the beam but are unable to improve all required parameters, e.g., emittance. Technical advancements on those elements would be needed to match the needs of laser-driven particles. The use of beam tailoring apertures and magnets might fail due to very stringent characteristics in terms of beam pointing stability and reliability. The high current of laser-driven electron beams enables them to be used as a driver for a free electron laser, but unfortunately the spectral characteristics of the radiation are not comparable with those obtained using conventional accelerators. As a consequence, much effort will be needed on both, source side and transport system, in order to achieve laser-driven beamlines that can compete with conventional accelerator ones.

\section{ACKNOWLEDGMENTS}

The authors acknowledge the fruitful discussions with the SPARC group and the support of ELI-PP (FP7 Contract No. 212105), CRISP (FP7 Contract No. 283745), and MIUR-SPARX Funding.

\footnotetext{
${ }^{1}$ The Future of Accelerator Physics, edited by T. Tajima (AIP, New York, 1996).

${ }^{2}$ See http://www.cern.ch for details about the Large Hadron Collider, its main characteristics and last achievements.

${ }^{3}$ E. Esarey et al., Rev. Mod. Phys. 81(3), 1229 (2009).

${ }^{4}$ C. G. R. Geddes et al., Nature 431, 535 (2004); S. P. D. Mangles et al., ibid. 431, 538 (2004); J. Faure et al., ibid. 431, 541 (2004).

${ }^{5}$ W. P. Leemans et al., Nat. Phys. 2, 696 (2006).

${ }^{6}$ S. Gordienko and A. Pukhov, Phys. Plasmas 12, 043109 (2005).

${ }^{7}$ W. Lu et al., Phys. Rev. ST Accel. Beams 10, 061301 (2007).

${ }^{8}$ E. Brunetti et al., Phys. Rev. Lett. 105, 215007 (2010).

${ }^{9}$ C. M. S. Sears et al., Phys. Rev. ST Accel. Beams 13, 092803 (2010).

${ }^{10}$ C. Rechatin et al., Phys. Rev. Lett. 102, 164801 (2009).

${ }^{11}$ A. J. Gonsalves et al., Nat. Phys. 7, 862 (2011).

${ }^{12} \mathrm{M}$. Reiser, Theory and Design of Charged Particle Beams, 2nd ed. (Wiley VCH, Weinheim), p. 105.

${ }^{13}$ See http://ilil.ino.it/flame/index.html for details about the main characteristics and last achievements of the 200 TW Ti:Sa FLAME laser system installed at INFN in Frascati.

${ }^{14}$ C. Benedetti, AIP Conf. Proc. 1209, 11-14 (2010).

${ }^{15}$ C. Benedetti et al., IEEE Trans. Plasma Sci. 36(4), 1790-S98 (2008); C. Benedetti et al., Nucl. Instrum. Methods Phys. Res. A 608, S94-S98 (2009).

${ }^{16}$ L. M. Young, private communication (2009).

${ }^{17}$ L. M. Young, "PARMELA," Los Alamos National Laboratory Report LAUR-96-1835 (Revised April. 22, 2003).

${ }^{18}$ Advances in Solid-State Lasers: Development and Applications, edited by M. Grishin (InTech, 2010), p. 600.
} 
${ }^{19}$ M. Ferrario et al., Proceedings of FEL08, Gyeongju, Korea, TUPPH048 (JACoW, 2008), p. 359.

${ }^{20}$ E. D. Courant, Part. Accel. 2, 117 (1972).

${ }^{21}$ K. R. Crandall and D. P. Rusthoi, "TRACE 3D documentation," LA-UR97-886, 1997.

${ }^{22}$ S. Becker et al., Phys. Rev. ST Accel. Beams 12, 102801 (2009).

${ }^{23} \mathrm{~J}$. B. Rosenzweig et al., "RF and magnetic measurements on the SPARC photoinjector and solenoid at UCLA, in PAC05 Proceedings, Knoxville, TN (JACoW, 2005), p. 2626.

${ }^{24}$ J. Schmerge, "LCLS gun solenoid design considerations," LCLS-TN-05$14,2005$.
${ }^{25}$ S. A. Kahn et al., "HTS development for 30-50 T final muon cooling solenoids," in PAC09 Proceedings, Vancouver, BC, Canada, 2009.

${ }^{26}$ S. Reiche, Genesis 1.3 User Manual, Available at: http://genesis.web. psi.ch/.

${ }^{27}$ L. Palumbo et al., "SPARX-FEL Technical Design Report," (2009); Available at: http://www.sparx-fel.it.

${ }^{28}$ S. Kneip et al., Phys. Rev. ST Accel. Beams 15, 021302 (2012).

${ }^{29}$ S. Fritzler et al., Phys. Rev. Lett. 92(16), 165006 (2004).

${ }^{30}$ J. Osterhoff et al., Phys. Rev. Lett. 101, 085002 (2008).

${ }^{31}$ M. Fuchs et al., Nat. Phys. 5, 1404 (2009).

${ }^{32}$ K. Floettmann, Phys. Rev. ST Accel. Beams 6, 034202 (2003). 